\title{
Extrahepatic diseases and NAFLD: The triangular relationship between NAFLD, type 2-diabetes and dysbiosis
}

Eleonora Scorletti ${ }^{1,2}$, Christopher D. Byrne ${ }^{1,2}$,

${ }^{1}$ Nutrition and Metabolism, Faculty of Medicine, University of Southampton, Southampton, UK

${ }^{2}$ Southampton National Institute for Health Research Biomedical Research Centre, University

Hospital Southampton, UK

Key words : non-alcoholic fatty liver disease, gut microbiota, dysbiosis, type 2 diabetes, insulin resistance.

Conflict of Interests: nothing to declare

Address for corres pondence:

Prof. Christopher D. Byrne

Nutrition and Metabolism, Faculty of Medicine

University of Southampton

Southampton SO16 6YD, UK

E-mail: cdtb@ soton.ac.uk

Phone: +442381208818 


\section{Abstract $=\mathbf{2 8 8}$ words}

Non-alcoholic fatty liver disease (NAFLD) encompasses a spectrum of liver diseases from simple steatosis with hepatic lipid accumulation to end stage liver disease with decompensated cirrhosis, liver failure and hepatocellular carcinoma. Recent data from the USA showed that in 2013, NAFLD was the second most frequent indication for liver transplantation behind Hepatitis C. Since there are now effective treatments for Hepatitis C and there is currently no licensed treatment for NAFLD, it has been predicted that over the next 10-15 years, NAFLD will replace Hepatitis $\mathrm{C}$ as the most frequent indication for liver transplantation. Besides, increasing the risk of hepatocellular carcinoma and end stage liver disease, recently it has become clear that NAFLD also increases risk of extra-hepatic diseases such as type 2 diabetes (T2DM), cardiovascular (CVD), card iac diseases and chronic kidney disease, to name but a few. Of each of these extra-hepatic diseases, the evidence to date suggests that NAFLD is a strong risk factor for T2DM. When NAFLD occurs in combination with obesity and insulin resistance (as it frequently does), there is a marked increase in risk of incident T2DM with possible synergism occurring between liver fat accumulation, insulin resistance and obesity, to further increase risk of development of T2DM. Thus, there is a reciprocal relationship between NAFLD as a risk factor for T2DM, and T2DM as a risk factor for liver disease progression in NAFLD. Moreover, recent evidence now points to the importance of perturbation of the intestinal microbiota (dysbiosis) in both T2DM and NAFLD. Consequently, there is a triangular relationship between dysbiosis-T2DM-NAFLD. This review will focus on T2DM as a key extra-hepatic complication of NAFLD and will describe and discuss the triangular relationship between dysbiosis-T2DM-NAFLD and the factors and potential mechanisms underpinning this relationship. 


\section{Introduction}

Non-alcoholic fatty liver disease (NAFLD) is the first stage of chronic liver disease and is a major cause of liver-related morbidity and mortality. NAFLD begins with lipid accumulation in the hepatocytes, it can progress to liver inflammation with activation of hepatic stellate cells (non-alcoholic steatohepatitis-NASH), subsequently leading to fibrosis with hepatic collagen deposition, and ultimately to hepatocellular carcinoma. The development of hepatic fibrosis occurs in $40-50 \%$ of patients with NASH and current estimates are that approximately $30-40 \%$ of people with NAFLD develop NASH [1]. There is growing evidence that NAFLD is a multisystem disease, affecting several extra-hepatic organs and regulatory pathways. Since NAFLD has become the predominant cause of chronic liver disease in many parts of the world, NAFLD is also potentially contributing to an important burden of extra-hepatic chronic complications such as T2DM [2;3].

NAFLD has a multifactorial pathophysiology involving genetic (PNPLA3 I148M polymorphism) [4] and metabolic factors, such as sedentary lifestyle, increased intake of energy-rich foods, malnutrition characterized by an imbalanced intake of nutrients (e.g. high fat and high proteins diet, low fiber intake, high fructose intake) [5], altered gut microbiota composition (dysbiosis) [6], obesity, insulin resistance and T2DM [7]. The interaction between diet, host and gut microbiota can modulate inflammatory signalling pathways that in turn cause obesity, NAFLD and insulin resistance. The number of bacterial cells present in the mammalian gastrointestinal tract increases according to its $\mathrm{pH}$. There are approximately $1 \times 10^{3}$ bacteria per gram of contents in the stomach and duodenum where the $\mathrm{pH}$ ranges from 1.5 to 4 . In the jejunum and ileum, there are approximately $1 \times 10^{4}-1 \times 10^{7}$ bacteria, culminating in the colon $(\mathrm{pH}=5.5-7)$ with $1 \times 10^{11}-1 \times 10^{12}$ bacteria. [8]

Recent data from animal studies has shown that the gut microbiota contributes to the pathogenesis of NAFLD via multiple mechanisms including regulation of energy homeostasis [9] and metabolic endotoxemia [10]. 
Dysbiosis favors the overgrowth of Gram-negative bacteria, leading to an increased production of lipopolysaccharide (LPS) from the Gram-negative cell walls. Cani et al. have identified LPS as a trigger factor for insulin resistance, obesity and diabetes; they also demonstrated that LPS causes metabolic endotoxemia and inflammation [11].

This review will describe and discuss the triangular relationship between dysbiosis-T2DMNAFLD and the factors and potential mechanisms underpinning this relationship (Figure 1). 


\section{Gut dysbiosis and NAFLD}

The prevalence of NAFLD in the general population is estimated at 20-30\%; however, this prevalence increases up to $75-100 \%$ in presence of obesity and T2DM. Currently, one third of the global population is obese/overweight increasing the risk of developing NAFLD [12]. There are different aspects influencing the aetiology of obesity such as genetic, physiological and lifestyle factors.

Recent studies have shown a functional relationship between gut microbiota composition and obesity [13]. The human gut microbiota is characterized by five bacterial Phyla (Firmicutes, Bacteroidetes, Actinobacteria and Verrucomicrobia) and one Archea (Euryarchaeota). There are endogenous (dietary habits, fibre intake, lifestyle, drugs) and exogenous (bacterial mucosal interactions, intestinal $\mathrm{pH}$, and immune response) factors that can influence the equilibrium of the gut microbiota[14] .

Several human and animal studies have shown a specific change in the gut microbiota composition in obese people with a reduction in Bacteroidetes phylum and increase in Firmicutes phylum $[15 ; 16]$. Ley et al. studied the intestinal microbiota of genetically obese ob/ob mice, lean ob/+ and wild-type siblings fed with the same polysaccharide-rich diet. They showed that the genetically obese mice had a $50 \%$ reduction of Bactero idetes phylum and a proportional increase in Firmicutes phylum compared to lean sibling mice, despite beingfed with the same diet [17]. Similar changes in gut microbiota have been found in human studies. Interestingly, Turnbaugh et al. showed that the colonization of germ-free mice with an 'obese gut microbiota' results in a significantly greater increase in total body fat than colonization with a 'lean microbiota'. With this study they showed that obese microbiome (the genomic content of the gut microbiota) had an increased capacity to harvest energy from food and therefore it is an additional contributing factor to the pathophysiology of obesity [18]. 
Healthy gut bacteria ferment complex carbohydrates into short-chain fatty acids (SCFAs), (primarily acetate, propionate, and butyrate) and make them absorbable by the intestinal epithelium [19]. Butyrate is an important energy source for the colonocytes, while acetate and propionate are primarily absorbed and extracted by the liver where they are used as substrates for lipogenesis and gluconeogenesis [20]. SCFAs are necessary for the growth of healthy bacteria, the regulation of intestinal $\mathrm{pH}$, and the maintenance of the gut bacteria health equilibrium. All these SCAFs functions have beneficial effects on NAFLD [21]. Moreover, SCFAs production is important as SCFAs have a strong capacity to downregulate inflammatory processes. SCFAs bind to and stimulate the chemoattractant receptor GPR43 on neutrophils, that is necessary for the resolution of inflammatory responses [22].

Qin et al. have shown that, in patients with cirrhosis, the composition of the gut microbiota is characterized by low abundance in butyrate-producing bacteria compared with healthy control group subjects, in which there is a "healthy gene-rich" microbiome [23]. These authors identified several bacteria as part of the "healthy gene-rich" microbiome, including Faecalibacterium prausnitzii, which has anti-inflammatory properties, and Coprococcus comes, which contributes to gut health through butyrate production [23]. In a different study, Chen et al. used pyrosequencing of the $16 \mathrm{~S}$ rRNA V3 region and real-time qPCR to characterize the gut microbiota of patients with cirrhosis. They found markedly high levels of several Gram negative bacteria, such as Proteobacteria and Fusobacteria [24]. Similarly, van Best and colleagues showed that, in patients with NAFLD, Proteobacteria, Enterobacteriaceae (Escherichia) and Ruminococcaceae (Faecalibacterium) were abundant compared with obese and healthy subjects [25]. In a recent study on fifty-seven patients with biopsy-proven NAFLD, Boursier et al. identified that Bacteroides were independently associated with NASH and that Ruminococcus are associated with significant fibrosis [26] . In addition, Zhu et al. showed that there are ethanol-producing bacteria, such as Escherichia$C$., that are capable of aggravating liver condition. These bacteria constantly produce alcohol 
and thus continuously supply reactive oxygen species to the liver, causing liver inflammation and NASH [27].

\section{Dysbiosis and adipose tissue triglyceride regulation}

In presence of high energy intake and low energy expenditure, adipose tissue is overloaded by an excess of energy stored as lipid (triacylglycerol). The ability of expansion of the peripheral adipose tissue provides a temporary buffering capacity that protects the liver from an excessive flux of free fatty acid (FFA) which would otherwise promote hepatic lipid accumulation. If this protective mechanism fails, excessive FFA causes hepatic/peripheral insulin resistance, hepatic inflammation and in turn increases the risk of progressive liver disease with fibrosis, cirrhosis and hepatocellular carcinoma [28].

As a consequence of dysbiosis, gut microbiota can suppress the intestinal epithelial expression of the fasting-induced adipose factor (Fiaf), also known as angiopoietin-like protein-4 (ANGPTL4), that is an inhibitor of circulating lipoprotein lipase (LPL) [14]. ANGPTL4 is a protein secreted by the liver and adipose tissue that plays an important role in lipid metabolism and glucose homeostasis. LPL is the main rate-limiting enzyme in the lipid metabolism pathway. It primarily catalyses the hydrolysis of triglycerides into free fatty acids and monoacylglycerol [29].

Reduced level of ANGPTL4 fails to inhibit LPL activity and therefore there is an increasein FFA flux from the adipose tissue to the liver that increases the availability of long-chain fatty acyl-CoAs for hepatic lipid accumulation. LPL overexpression in the liver has been associated with liver triglyceride accumulation and NAFLD [30]. 
Consistently, a mice study has showed that supplementation with probiotic bacteria Lactobacillus paracasei was associated with a beneficial effect on ANGPTL4, inhibiting LPL activity and improving lipid metabolism [31].

A further effect of gut microbiota on lipid metabolism concerns the regulation of dietary choline in the intestine. Choline is an essential nutrient and it is mostly obtained from food such as red meat and eggs. Choline is essential for neurotransmitter synthesis, methyl-group metabolism, cell- membrane structure and signalling, lipid metabolism and synthesis of verylow-density lipoprotein in the liver [32]. Moreover, choline is metabolized in the liver to phosphatidylcholine, which is necessary for the packaging and export of triglycerides in very low density lipoproteins. The conversion of dietary choline to methylamines by gut dysbiosis reduces the bioavailability of choline in the intestine and mimics the effect of choline-deficient diets, causing a predisposition to impaired glucose homeostasis [33].

\section{Gut dysbiosis, NAFLD and type 2 diabetes}

Recent evidence shows that gut dysbiosis also plays a key role in the pathogenesis and progression of NAFLD and T2DM, regulating several inflammatory pathways (Table 1) [34]. In a human study, Larsen et al. assessed the differences in gut microbiota composition in 36 men with and without T2DM. They found that in patients with T2DM there was an increase in gram-negative bacteria belonging to the Bacteroidetes and Proteobacteria compared with the control group [35].

Two similar human studies showed significant correlations of specific gut bacteria and their genes with T2DM. The first was conducted in China; Qin et al mapped the metagenome sequences to known metagenome data sets [36]. Their analyses indicated a decrease in butyrate-producing bacteria (such as Roseburia intestinalis) and an increase in some opportunistic bacteria, as well as enrichment in pathways responsible for the oxidative stress in T2DM patients, compared with people without diabetes. Qin also introduced the concept 
of 'functional dysbiosis', explaining that there was an association with loss of certain functional bacteria such as butyrate producing-bacteria, rather than loss of specific gut microbiota directly related to T2DM. The second study was undertaken in European women with normal, impaired or poor glucose control. Karlsson et al used a metagenome-wide association study and developed a mathematical model based on metagenomics clusters (MGCs), which allowed DNA that had not previously been sequenced to be included in the analysis that identified T2DM [37]. Although Qin and Karlsson used different sequencing techniques to characterize the gut microbiota in T2DM patients, the results showed significant correlations of specific gut microbiome and T2DM. Both studies suggested that functional dysbiosis might reflect changes in intestinal environment that in turn favours the growth of certain Gram-negative bacteria linked with T2DM. In addition, both the aforementioned studies showed lower concentrations of Roseburia intestinalis and Faecalibacterium prausnittzii and higher levels of Lactobacillus gasseri, Streptococcus mutans, and Clostridiales in patients with T2DM.

Gut barrier integrity is another important physiological protection against endotoxins produced by Gram-negative bacteria. Several studies have shown that high-fat diet reduces Bifidobacterium phylum with a secondary increase of lipopolysaccharide (LPS) production and inflammatory activity [38]. In the presence of dysbiosis, there is an increased production of endotoxins from the Gram- negative bacteria that can damage the intestinal barrier. These endotoxins are then released in the blood stream causing a subclinical elevation in circulating levels of LPS [39]. This metabolic endotoxiemia is characterized by a low-grade metabolic inflammation affecting different pathways linked with metabolic syndrome, NAFLD and insulin resistance.

The degradation of the intestinal mucus layer can lead to intestinal inflammation and reduced function of L-cells and Paneth cells in the intestine. Akkermantia muciniphila is a mucindegrading bacterium that resides in the mucus layer of the intestine. It is the dominant 
bacterium in the human intestine, representing 3-5\% of the microbial community in healthy subjects. Although Akkermantia muciniphila is a Gram negative bacterium that contains LPS, the gut colonization of these bacteria has a protective role in metabolic endotoxiemia [40]. There is an inverse correlation between the abundance of Akkermantia muciniphila and presence of obesity and T2DM.

In a study in mice, Everard and colleagues demonstrated that the administration of alive Akkermantia muciniphila restores the intestine mucus layer reducing the metabolic endotoxiemia [41]. Paneth cells are part of the epithelium in the small intestine. They produce several antimicrobial peptides for innate immunity such as the regenerating isletderived III (RegIII- $\alpha$ RegIII- $\beta$ and RegIII- $\gamma$ ) proteins which belong to the family of C-type lectins [42]. RegIII- $\gamma$ has a specific antibacterial effect and administration of alive Akkermantia muciniphila or Bifidobacterium breve effectively induces REGIII production and specifically RegIII- $\gamma$ production protecting the intestine barrier [43]. Moreover, Akkermantia muciniphila is associated with higher L-cell activity and consequent increased production of GLP-1 and GLP-2. Therefore, administration of Akkermantia muciniphila may improve glucose tolerance and decreased endogenous hepatic glucose production via an incretin- mediated effect.

Recent evidence shows that one possible antidiabetic effect of metformin is the manipulation of the gut microbiota, favouring the growth of Akkermantia muciniphila and reducing gramnegative bacteria responsible for producing endotoxins [44]. Mounting evidence further suggests that one of the mechanisms of glucose-lowering effect of metformin is the modulation of gut microbiota. Metformin-induced effects in the intestine may also alter the bile acid pool to stimulate GLP-1 secretion and thereby affect risk of type 2 diabetes. The increase in the bile acid pool may then stimulate bile acid receptors on the L cell, causing an increase in GLP-1 secretion via mitochondrial oxidative phosphorylation and calcium influx. In addition, a study has shown that metformin protects against fructose-induced steatosis [45]. 
Spruss et al found that in mice with NAFLD, metformin reduces the translocation of gut bacteria and bacterial endotoxins, by protecting mice from the fructose-induced loss of tight junction proteins and zonula occludens 1 (Figure 2) [46;47]. 


\section{Dysbiosis and insulin resistance}

Both NAFLD and T2DM are characterized by insulin resistance and a state of chronic lowgrade inflammation with endotoxiemia, abnormal expression and production of hepatokines, interleukins and adipokynes. Insulin resistance is caused by several factors such as lifestyle, nutritional habits, genetic determinants, and ectopic lipid accumulation in the liver and muscles. Recent evidence shows that dysbiosis may also influence insulin resistance [48]. Interestingly, there is an increasing body of scholarship showing that a state of chronic inflammation and a pathological activation of the innate immune system causing an increased production of cytokines and/or adipokines may affect the regulation of insulin action [49].

The physiological effect of insulin begins with the activation of the insulin receptor (IR) tyrosine kinase, which in turn promotes tyrosine kinase phosphorylation of insulin receptor substrates (IRS-1 and IRS-2). Phosphorylation of IRS-1 and -2 activate phosphatidylinositol3-OH kinase (PI3-K), which increases serine phosphorylation of Akt. The insulin signalling pathway may be disrupted by several mechanisms associated with, on one hand, metabolic inflammation as a consequence of LPS and endotoxin production, and, on the other, increased level of diacylglycerols (DAGs) and ceramides with a high fat diet. The disruption of insulin signal occurs via serine phosphorylation of IRS and decreased tyrosine phosphorylation of IRS. Circulating LPS or bacterial endotoxins stimulates inflammatory cytokine production and increases the activation of Toll-like Receptor (TLR) 2 and 4. The activation of TLRs increases the expression of several cytokines such as TNF- $\alpha$ and IL-6 involved in insulin signalling. c-Jun amino-terminal kinase (JNK) and the inhibitor of nuclear factor- $\kappa \mathrm{B}(\mathrm{NF}-\kappa \mathrm{B})$ kinase- $\beta(\mathrm{IKK}-\beta)$ are activated upon the exposure to cytokines such as TNF- $\alpha$ and IL-6, free fatty acids and endoplasmic reticulum stress [50]. The activation of the liver specific JNK promotes hepatic steatosis associated with impaired expression of liver key lipogenic enzymes and impairs insulin signalling via serine phosphorylation of IRS1. 
The activation of IKK reduces the phosphorylation of Akt 2 and increases ceramide synthesis. The impaired phosphorylation of Akt2 reduces insulin action and insulin suppression of hepatic gluconeogenesis [48].

Within hepatocytes, long-chain fatty acids (LCFAs) are esterified with glycerol-3-phosphate (derived from glycolysis) to form mono-acylglycerols, di-acylglycerols (DAG) and triacylglycerols (TAG). Lipid synthesis may increase production of intermediate lipids, such as DAG, di-palmitoyl phosphatic acid (Di-P PA) and other lipid products, such as ceramides. The increased production of these lipid products (particularly DAGs) is very important in causing 'resistance' within the hepatic insulin-signalling pathway [51], promoting hepatic inflammation and increasing risk of progressive liver disease that occurs with NASH. Ceramide accumulation in the liver leads to an impairment of insulin action reducing Akt2 activation and to the activation of the PKC $\zeta$ iso form. In addition, the increased levels of DAG in the liver can contribute to protein kinase $\mathrm{C} \varepsilon(\mathrm{PKC} \varepsilon)$ activation and translocation to the cell membrane, which results in inhibition of insulin signalling via inhibition of insulin receptor kinase activity. Gut microbiota can play a significant role in DAG production. Gut dysbiosis can lower intestinal $\mathrm{pH}$ and increase the production of secondary bile acids. The combination of low $\mathrm{pH}(<7)$ and secondary bile acids (mainly deoxycholic acid) favors the degradation of phosphatidylcholine by bacteria expressing phospholipase C (e.g. $C$. bifermentans and E. coli.) resulting in an increased DAG production and promoting hepatic inflammation, liver fat accumulation and impaired insulin signalling. There is also evidence that increased production of secondary bile acids by the gut bacteria may activate PKC thus impairing insulin signalling [52]. 


\section{Conclusions}

Currently, there are limited human studies describing the association between gut microbiota, T2DM and NAFLD. In patients with NAFLD, there is clear evidence that diet can cause specific changes in the gut microbiota which affect the aforementioned association. By contrast, there are very limited data describing NAFLD-specific changes in gut microbiota that are independent of diet and obesity. Some mechanisms that are relative to a putative link between dysbiosis, type 2 diabetes and NAFLD may include increased intestinal permeability, low grade inflammation with endotoxemia, changes in the production of SCFAs with reduction of butyrate producing bacteria, alterations in choline metabolism, and/or effects on the secretion of gut hormones such as GLP-1 and GLP-2. The similitude between bacteria that cause NAFLD and bacteria that cause T2DM could be the key to understand the triangular relationship between dysbiosis-T2DM-NAFLD. These interactions are still poorly understood, although metagenomic studies have provided an enormous amount of data concerning the characterization of microbiota from different parts of the host's body in different conditions. High quality research clinical trials are needed to test whether improving dysbiosis ameliorates NAFLD and T2DM.

Moreover, the newly described effects of metformin treatment on gut microbiota require further investigation to broaden our understanding of the molecular mechanisms of metformin action on the intestinal bacteria and to comprehend fully the dysbiotic changes in people with T2DM.

Finally, clinical research trials are urgently needed to test whether pre-probiotics or synbiotics have a beneficial effect on the gut microbiota of patients with NAFLD and type 2 diabetes. 


\section{Conflict of interest}

All authors have nothing to disclose.

\section{Acknowledgements}

This work was supported by the National Institute of Health Research (NIHR) through the NIHR Southampton NIHR Biomedical Research Unit in Nutrition (and Lifestyle), by Diabetes UK, and by the Parnell Diabetes Trust. CDB, PCC, and ES are supported in part by the National Institute for Health Research Southampton Biomedical Research Centre. 
1 Wang X, Li J, Riaz DR, Shi G, Liu C, Dai Y: Outcomes of liver transplantation for nonalcoholic steatohepatitis: a systematic review and meta-analysis. Clin Gastroenterol Hepatol 2014;12:394-402.

2 Byme CD, Targher G: Ectopic fat, insulin resistance, and nonalcoholic fatty liver disease: implications for cardiovascular disease. Arterioscler Thromb Vasc Biol 2014;34:1155-1161.

3 Bhatia LS, Curzen NP, Calder PC, Byme CD: Non-alcoholic fatty liver disease: a new and important cardiovascular risk factor? Eur Heart J 2012.

4 Scorletti E, West AL, Bhatia L, Hoile SP, McCormick KG, Burdge GC, Lillycrop KA, Clough GF, Calder PC, Byrne CD: Treating liver fat and serum triglyceride levels in NAFLD, effects of PNPLA3 and TM6SF2 genotypes: Results from the WELCOME trial. J Hepatol 2015;63:1476-1483.

Veena J, Muragundla A, Sidgiddi S, Subramaniam S: Non-alcoholic fatty liver disease: need for a balanced nutritional source. Br J Nutr 2014;112:1858-1872.

6

Henao-Mejia J, Elinav E, Thaiss CA, Licona-Limon P, Flavell RA: Role of the intestinal microbiome in liver disease. J Autoimmun 2013;46:66-73.

Targher G, Byrne CD: Clinical Review: Nonalcoholic fatty liver disease: a novel cardiometabolic risk factor for type 2 diabetes and its complications. J Clin Endocrinol Metab 2013;98:483-495.

8 Sekirov I, Russell SL, Antunes LC, Finlay BB: Gut microbiota in health and disease. Physiol Rev 2010;90:859-904.

9

Turnbaugh PJ, Ley RE, Mahowald MA, Magrini V, Mardis ER, Gordon JI: An obesity-associated gut microbiome with increased capacity for energy harvest. Nature 2006;444:1027-1031.

Cani PD, Bibiloni R, Knauf C, Waget A, Neyrinck AM, Delzenne NM, Burcelin R: Changes in gut microbiota control metabolic endotoxemia-induced inflammation in high-fat diet-induced obesity and diabetes in mice. Diabetes 2008;57:1470-1481.

11 Cani PD, Possemiers S, Van de Wiele T, Guiot Y, Everard A, Rottier O, Geurts L, Naslain D, Neyrinck A, Lambert DM, Muccioli GG, Delzenne NM: Changes in gut microbiota control inflammation in obese mice through a mechanism involving GLP-2driven improvement of gut permeability. Gut 2009;58:1091-1103.

13 Angelakis E, Armougom F, Million M, Raoult D: The relationship between gut microbiota and weight gain in humans. Future Microbiol 2012;7:91-109. mutualism in the human intestine. Science 2005;307:1915-1920. 

in energy harvesting? Gut 2010;59:1589-1590.

Mehal WZ: The Gordian Knot of dysbiosis, obesity and NAFLD. Nat Rev Gastroenterol Hepatol 2013;10:637-644.

Ley RE, Backhed F, Turnbaugh P, Lozupone CA, Knight RD, Gordon JI: Obesity alters gut microbial ecology. Proc Natl Acad Sci U S A 2005;102:11070-11075.

Ley RE, Turnbaugh PJ, Klein S, Gordon JI: Microbial ecology: human gut microbes associated with obesity. Nature 2006;444:1022-1023.

den BG, van EK, Groen AK, Venema K, Reijngoud DJ, Bakker BM: The role of short-chain fatty acids in the interplay between diet, gut microbiota, and host energy metabolism. J Lipid Res 2013;54:2325-2340.

Utzschneider KM, Kratz M, Damman CJ, Hullar M: Mechanisms linking the gut microbiome and glucose metabolism. J Clin Endocrinol Metab 2016;jc20154251.

Endo H, Niioka M, Kobayashi N, Tanaka M, Watanabe T: Butyrate-producing probiotics reduce nonalcoholic fatty liver disease progression in rats: new insight into the probiotics for the gut-liver axis. PLoS One 2013;8:e63388.

Karaki S, Mitsui R, Hayashi H, Kato I, Sugiya H, Iwanaga T, Furness JB, Kuwahara A: Short-chain fatty acid receptor, GPR43, is expressed by enteroendocrine cells and mucosal mast cells in rat intestine. Cell Tissue Res 2006;324:353-360.

Qin N, Yang F, Li A, Prifti E, Chen Y, Shao L, Guo J, Le CE, Yao J, Wu L, Zhou J, Ni S, Liu L, Pons N, Batto JM, Kennedy SP, Leonard P, Yuan C, Ding W, Chen Y, Hu X, Zheng B, Qian G, Xu W, Ehrlich SD, Zheng S, Li L: Alterations of the human gut microbiome in liver cirrhosis. Nature 2014;513:59-64.

24 Chen Y, Yang F, Lu H, Wang B, Chen Y, Lei D, Wang Y, Thu B, Li L: Characterization of fecal microbial communities in patients with liver cirrhosis. Hepatology 2011;54:562-572.

van BN, Jansen PL, RensenSS: The gut microbiota of nonalcoholic fatty liver disease: current methods and their interpretation. Hepatol Int 2015;9:406-415.

Boursier J, Mueller O, Barret M, Machado M, Fizanne L, Araujo-Perez F, Guy CD, Seed PC, Rawls JF, David LA, Hunault G, Oberti F, Cales P, Diehl AM: The severity of nonalcoholic fatty liver disease is associated with gut dysbiosis and shift in the metabolic function of the gut microbiota. Hepatology 2016;63:764-775.

Characterization of gut microbiomes in nonalcoholic steatohepatitis (NASH) patients: a connection between endogenous alcohol and NASH. Hepatology 2013;57:601-609.

28 Byme CD: Ectopic fat, insulin resistance and non-alcoholic fatty liver disease. Proc Nutr Soc 2013;1-8.

29 Lichtenstein L, Berbee JF, van Dijk SJ, van Dijk KW, Bensadoun A, Kema IP, Voshol PJ, Muller M, Rensen PC, Kersten S: Angptl4 upregulates cholesterol synthesis 
in liver via inhibition of LPL- and HL-dependent hepatic cholesterol uptake. Arterioscler Thromb Vasc Biol 2007;27:2420-2427.

Zhang L, Zhang Z, Li Y, Liao S, Wu X, Chang Q, Liang B: Cholesterol induces lipoprotein lipase expression in a tree shrew (Tupaia belangeri chinensis) model of non-alcoholic fatty liver disease. Sci Rep 2015;5:15970.

Aronsson L, Huang Y, Parini P, Korach-Andre M, Hakanss on J, Gustafsson JA, Pettersson S, Arulampalam V, Rafter J: Decreased fat storage by Lactobacillus paracasei is associated with increased levels of angiopoietin-like 4 protein (ANGPTL4). PLoS One 2010;5.

Minahk C, Kim KW, Nelson R, Trigatti B, Lehner R, Vance DE: Conversion of low density lipoprotein-associated phosphatidylcholine to triacylglycerol by primary hepatocytes. J Biol Chem 2008;283:6449-6458.

Dumas ME, Barton RH, Toye A, Cloarec O, Blancher C, Rothwell A, Fearnside J, Tatoud R, Blanc V, Lindon JC, Mitchell SC, Holmes E, McCarthy MI, Scott J, Gauguier D, Nicholson JK: Metabolic profiling reveals a contribution of gut microbiota to fatty liver phenotype in insulin-resistant mice. Proc Natl Acad Sci U S A 2006;103:12511-12516.

Miele L, Giorgio V, Alberelli MA, De CE, Gasbarrini A, Grieco A: Impact of Gut Microbiota on Obesity, Diabetes, and Cardiovascular Disease Risk. Curr Cardiol Rep 2015;17:120.

Larsen N, Vogensen FK, van den Berg FW, Nielsen DS, Andreasen AS, Pedersen BK, Al-Soud WA, Sorensen SJ, Hansen LH, Jakobsen M: Gut microbiota in human adults with type 2 diabetes differs from non-diabetic adults. PLoS One 2010;5:e9085.

Qin J, Li Y, Cai Z, Li S, Zhu J, Zhang F, Liang S, Zhang W, Guan Y, Shen D, Peng Y, Zhang D, Jie Z, Wu W, Qin Y, Xue W, Li J, Han L, Lu D, Wu P, Dai Y, Sun X, Li Z, Tang A, Zhong S, Li X, Chen W, Xu R, Wang M, Feng Q, Gong M, Yu J, Zhang Y, Thang M, Hansen T, Sanchez G, Raes J, Falony G, Okuda S, Almeida M, LeChatelier E, Renault P, Pons N, Batto JM, Zhang Z, Chen H, Yang R, Zheng W, Li S, Yang H, Wang J, Ehrlich SD, Nielsen R, Pedersen O, Kristiansen K, Wang J: A metagenome-wide association study of gut microbiota in type 2 diabetes. Nature 2012;490:55-60.

Karlsson FH, Tremaroli V, Nookaew I, Bergstrom G, Behre CJ, Fagerberg B, Nielsen J, Backhed F: Gut metagenome in European women with normal, impaired and diabetic glucose control. Nature 2013;498:99-103.

Arslan N: Obesity, fatty liver disease and intestinal microbiota. World J Gastroenterol 2014;20:16452-16463. Fava F, Tuohy KM, Chabo C, Waget A, Delmee E, Cousin B, Sulpice T, Chamontin B, Ferrieres J, Tanti JF, Gibson GR, Casteilla L, Delzenne NM, Alessi MC, Burcelin R: Metabolic endotoxemia initiates obesity and insulin resistance. Diabetes 2007;56:1761 1772. 
Schneeberger M, Everard A, Gomez-Valades AG, Matamoros S, Ramirez S, Delzenne NM, Gomis R, Claret M, Cani PD: Akkermansia muciniphila inversely correlates with the onset of inflammation, altered adipose tissue metabolism and metabolic disorders during obesity in mice. Sci Rep 2015;5:16643.

Everard A, Belzer C, Geurts L, Ouwerkerk JP, Druart C, Bindels LB, Guiot Y, Derrien M, Muccioli GG, Delzenne NM, de Vos WM, Cani PD: Cross-talk between Akkermansia muciniphila and intestinal epithelium controls diet-induced obesity. Proc Natl Acad Sci U S A 2013;110:9066-9071. Rodenas CL, Kiyama H, Bercik P, Verdu EF: Differential induction of antimicrobial REGIII by the intestinal microbiota and Bifidobacterium breve NCC2950. Appl Environ Microbiol 2013;79:7745-7754.

Cani PD, Everard A: [Akkermansia muciniphila: a novel target controlling obesity, type 2 diabetes and inflammation?]. Med Sci (Paris) 2014;30:125-127. in the Akkermansia spp. population induced by metformin treatment improves glucose homeostasis in diet-induced obese mice. Gut 2014;63:727-735.

Napolitano A, Miller S, Nicholls AW, Baker D, Van HS, Thomas E, Rajpal D, Spivak A, Brown JR, Nunez DJ: Novel gut-based pharmacology of metformin in patients with type 2 diabetes mellitus. PLoS One 2014;9:e100778.

Miele L, Valenza V, La TG, Montalto M, Cammarota G, Ricci R, Masciana R, Forgione A, Gabrieli ML, Perotti G, Vecchio FM, Rapaccini G, Gasbarrini G, Day CP, Grieco A: Increased intestinal permeability and tight junction alterations in nonalcoholic fatty liver disease. Hepatology 2009;49:1877-1887. against the development of fructose-induced steatosis in mice: role of the intestinal barrier function. Lab Invest 2012;92:1020-1032.

Caricilli AM, Saad MJ: The role of gut microbiota on insulin resistance. Nutrients 2013;5:829-851.

49 Tilg H, Moschen AR: Inflammatory mechanisms in the regulation of insulin resistance. Mol Med 2008;14:222-231.

50 Samuel VT, Shulman GI: Mechanisms for insulin resistance: common threads and missing links. Cell 2012;148:852-871.

51 Perry RJ, Samuel VT, Petersen KF, Shulman GI: The role of hepatic lipids in hepatic insulin resistance and type 2 diabetes. Nature 2014;510:84-91.

52 Vulevic J, McCartney AL, Gee JM, Johnson IT, Gibson GR: Microbial species involved in production of 1,2-sn-diacylglycerol and effects of phosphatidylcholine on human fecal microbiota. Appl Environ Microbiol 2004;70:5659-5666. 
Figure 1. The triangular relationship between Obesity, NAFLD and type two diabetes.

The similitude between bacteria that cause NAFLD and bacteria that cause type 2 diabetes the key to understand the triangular relationship between dysbiosis-T2DM-NAFLD.

Figure 2. Role of gut dysbiosis in non-alcoholic fatty liver disease, type 2 diabetes and insulin resistance. 1) The altered Firmicutes/Bacteroidetes ratio is associated with obesity phenotype; 2) Gram - negative bacteria abundance and butyrate producing bacteria reduction can damage the intestinal wall causing translocation of endotoxins and LPS into the circulation; 3) Dysbiosis causes a reduction of ANGPTL4 with consequent increase in LPL activity and triglycerides storage in the adipose tissue; 4) The decrease in Akkermantia Muciniphila in the intestine causes a disruption of the mucus layer with reduced $\mathrm{L}$ cells and Paneth cells function.

Table 1. Lists of healthy and unhealthy bacteria associated to NAFLD and type 2 diabetes. Bacteria and their role on the pathogenesis of NAFLD and type 2 diabetes. 
Figure 1

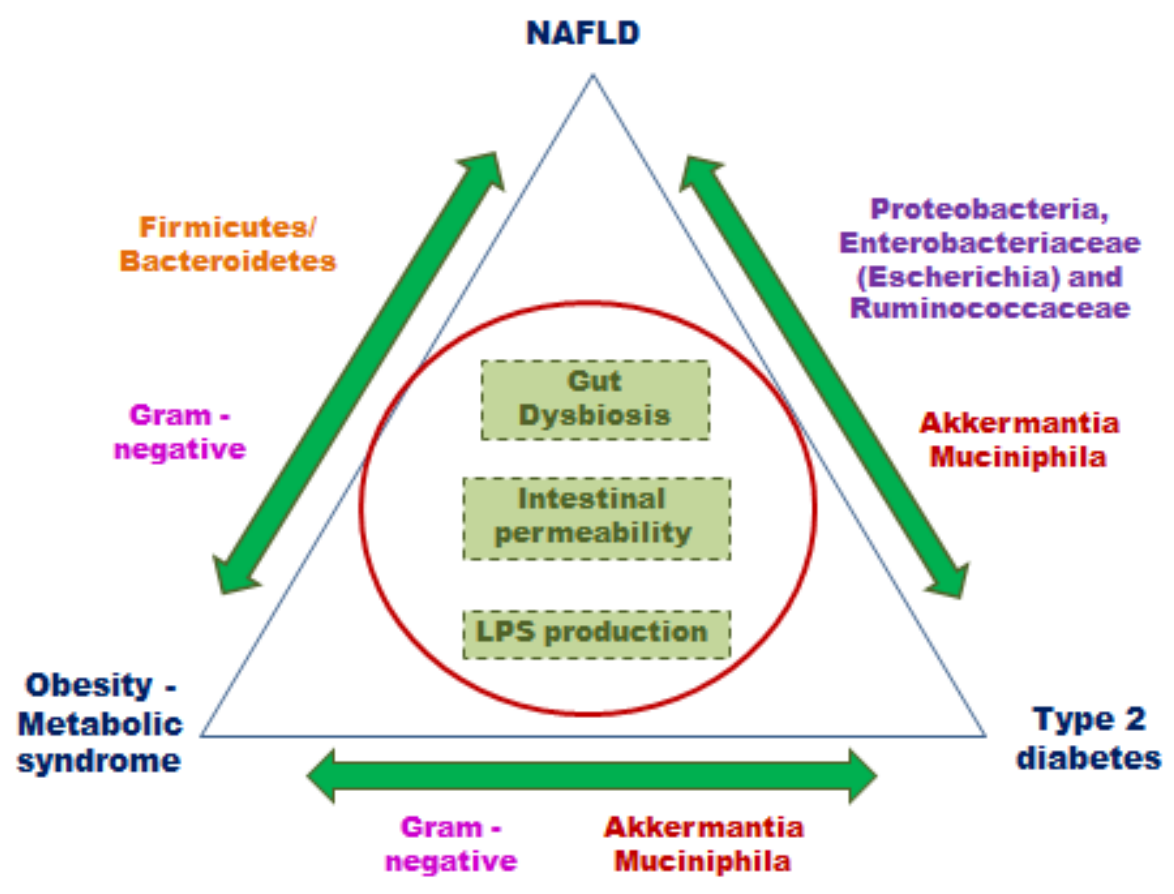


Figure 2

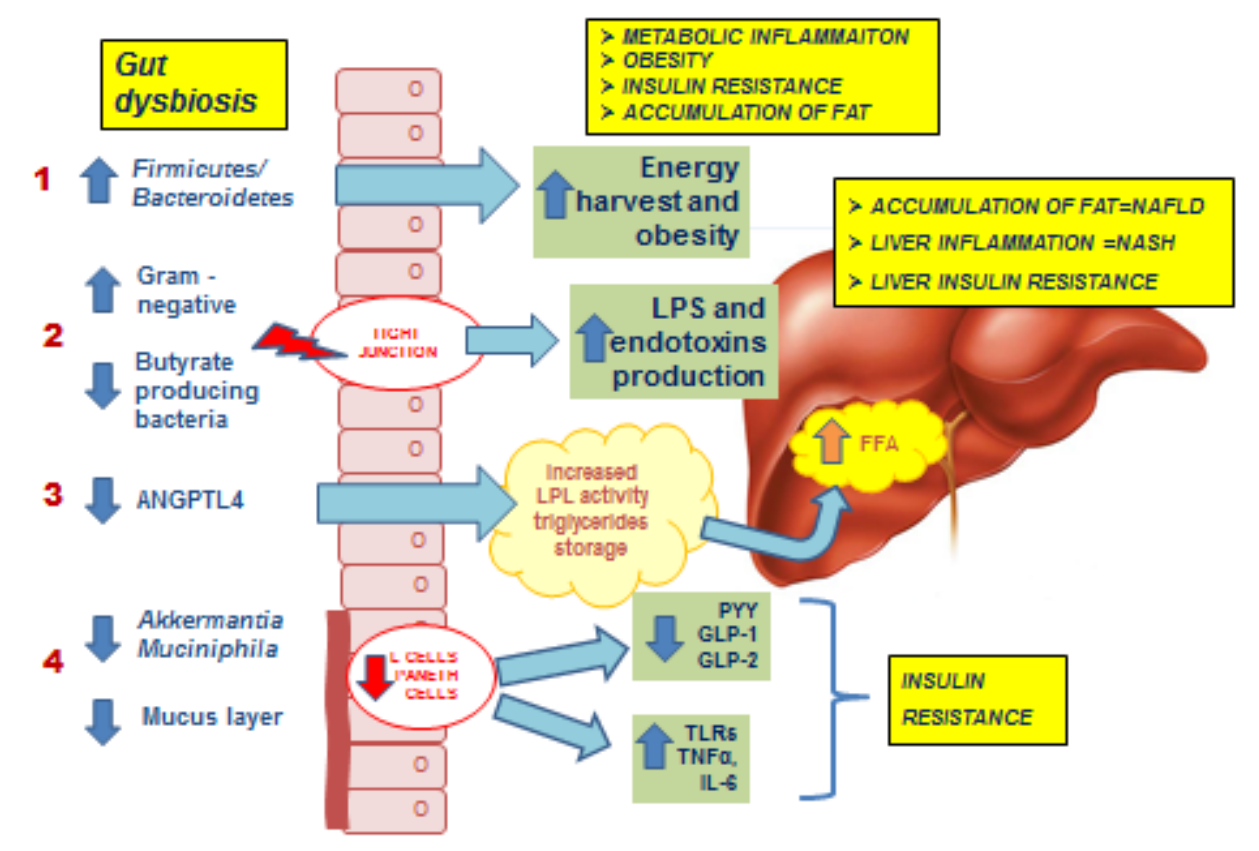




\section{Table 1}

\begin{tabular}{|c|c|c|}
\hline Intestinal becteria & Function & Cinical implicetion \\
\hline \multicolumn{3}{|l|}{ Healthy becteria } \\
\hline $\begin{array}{l}\text { Abelermontio } \\
\text { Muciniphile }\end{array}$ & Mucus layer protection & Decreased in type 2 diabetes \\
\hline Bifidobocterium breve & Mucus layer protection & Decreased in type 2 diabetes \\
\hline Foecolibocterium prousnitzii & Butyrate -producing & Decrease in NAFLD \\
\hline Coprococcus comes & Butyrate -producing & Decrease in NAFLD \\
\hline Roseburio intestinalis & Butyrate -producing & $\begin{array}{l}\text { Decrease in NAFLD and type } \\
2 \text { diabetes }\end{array}$ \\
\hline \multicolumn{3}{|l|}{ Dysbiosis } \\
\hline Proteobocterio & Endotoxins - LPS production & Increased in NAFLD \\
\hline Enterobocteriaceoe (Escherichio) & Endotoxins - LPS production & Increased in NAFLD \\
\hline Ruminococcoceoe (Foncolibocterium) & Endotoxins - LPS production & Increased in NAFLD \\
\hline Loctobocillus gosseri & Endotoxins - LPS production & Increased in type 2 diabetes \\
\hline Stroptococcus mutons, and Clostridioles & Endotoxins - LPS production & Increased in type 2 diabetes \\
\hline
\end{tabular}

